\title{
Vital capacities in acute and chronic airway obstruction: dependence on flow and volume histories
}

\author{
V. Brusasco*, R. Pellegrino**, J.R. Rodarte +
}

Vital capacities in acute and chronic airway obstruction: dependence on flow and volume histories. V. Brusasco, R. Pellegrino, J.R. Rodarte. CERS Journals Ltd 1997.

ABSTRACT: The aim of this study was to investigate whether measurements of vital capacity (VC) are affected by the direction of the manoeuvre (inspiratory vs expiratory) and by the rate of expiratory flow.

The study was performed on 25 individuals with chronic airway obstruction (CAO) and a forced expiratory volume in one second (FEV1) (expressed in standardized residuals (SR)) of $\mathbf{- 2 . 0 \pm 1 . 4} \mathrm{SD}$ (CAO group), and 10 asthmatic subjects with methacholine (MCh)-induced bronchoconstriction (FEV1 -2.3 \pm 1.02 SR) (MCh group). VCs were measured during fast inspiration following both slow (FIVCse) and forced (FIVCfe) expiration from end-tidal inspiration to residual volume (RV), and during slow (EVC) or forced (FVC) expiration from total lung capacity (TLC).

In the CAO group, FVC was the smallest volume $(3.75 \pm 1.03 \mathrm{~L})$ and significantly different from the other three estimates of VC; FIVCse $(4.03 \pm 0.91 \mathrm{~L})$ was the largest volume and significantly different from FVC and FIVCfe $(3.83 \pm 0.98 \mathrm{~L})$. In the MCh group, FVC $(4.16 \pm 0.94 \mathrm{~L})$ and $\mathrm{EVC}(4.19 \pm 0.89 \mathrm{~L})$ were the largest volumes, although only the difference between FVC and FIVCfe $(3.76 \pm 0.81 \mathrm{~L})$ reached statistical significance.

These data suggest that both flow and volume histories contribute to decreased vital capacities during bronchoconstriction. However, whereas increasing expiratory flow always tends to decrease vital capacity, the volume history of full inflation has different effects in chronic and acute bronchoconstriction, probably due to different effects on airway calibre. These results stress the importance of using standardized manoeuvres in order to obtain comparable values of vital capacity. Eur Respir J 1997; 10: 1320-1320.
*Cattedra di Fisiopatologia Respiratoria, Dipartimento di Scienze Motorie e Riabilitative, Università di Genova, Genova, Italy. **Servizio di Fisiopatologia Respiratoria, Azienda Ospedaliera S. Croce e Carle, Cuneo, Italy. +Pulmonary Section, Baylor College of Medicine, Houston, TX, USA.

Correspondence: V. Brusasco

Facoltà di Medicina e Chirurgia Università di Genova

Largo R. Benzi 10

16132 Genova

Italy

Keywords: Bronchoconstriction

deep inhalation

flow limitation

flow-volume curve

Received: June 141996

Accepted after revision February 101997

Supported by a Grant from MURST, Rome, Italy.
Vital capacity (VC) is defined as the maximum amount of air that can be mobilized with a single expiratory or inspiratory manoeuvre, i.e. the difference between total lung capacity (TLC) and residual volume (RV) [1, 2]. Hence, the size of VC depends on the determinants both of TLC and RV. In patients with airway obstruction, dynamic factors (flow limitation, airway closure) are determinants of RV [3, 4]. Therefore, it can be hypothesized that factors influencing airway calibre may also influence RV and, by inference, VC.

A previous volume history of deep inhalation may cause changes in airway calibre, the direction and magnitude of which depend on the site and the mechanism of airway obstruction [5-10]. Moreover, the RV attained after a forced expiration changes according to the direction and the magnitude of the bronchomotor effect of deep inhalation $[11,12]$. Based on these data, it may be expected that the size of $\mathrm{VC}$ measured from expiratory or inspiratory manoeuvres would differ. Furthermore, under conditions where RV is determined by dynamic factors occurring in the airways, it might be expected that changing expiratory flow would also affect the measurement of VC.
The aim of this study was to investigate the extent to which flow and volume histories may affect VC measurements. To this end, VC values obtained with expiratory or inspiratory manoeuvres and with inspiratory manoeuvres preceded by forced or slow exhalations to RV were compared.

\section{Methods}

\section{Subjects}

Two groups of subjects, whose anthropometric and pulmonary function data are presented in table 1, participated in the study after giving written informed consent.

The first group comprised 25 individuals with chronic airway obstruction (CAO group), as assessed by a forced expiratory volume in one second to forced vital capacity (FEV1/FVC) ratio below the normal range, and a FEV $1<80 \%$ of predicted [2]. According to the criteria of the American Thoracic Society (ATS) [13], 12 subjects 
Table 1. - Anthropometric and pulmonary function data under control conditions

\begin{tabular}{lccc}
\hline & CAO group & p-value & MCh group \\
\hline Subjects n & 25 & - & 10 \\
Age yrs & $47 \pm 16$ & $<0.01$ & $28 \pm 7$ \\
Height cm & $167 \pm 7$ & NS & $171 \pm 11$ \\
FEV1 L & $2.31 \pm 0.86$ & $<0.001$ & $3.38 \pm 1.08$ \\
$\quad$ SR & $-2.0 \pm 1.4$ & $<0.001$ & $0.1 \pm 1.2$ \\
FEV 1/FVC & $60 \pm 13$ & $<0.001$ & $81 \pm 8$ \\
RV L & $3.13 \pm 1.23$ & $<0.01$ & $1.87 \pm 0.43$ \\
SR & $2.9 \pm 2.3$ & $<0.01$ & $0.7 \pm 0.8$ \\
TLC L & $6.99 \pm 1.51$ & NS & $6.35 \pm 1.28$ \\
$\quad$ SR & $1.4 \pm 1.3$ & $<0.02$ & $0.2 \pm 0.7$ \\
\hline
\end{tabular}

Data are presented as mean \pm SD. CAO: chronic airflow obstruction; MCh group: asthmatics (before inhaling methacholine); FEV1: forced expiratory volume in one second; FVC: forced vital capacity; RV: residual volume; TLC: total lung capacity; SR: standardized residual; NS: nonsignificant.

had a clinical history of chronic bronchitis and 13 of bronchial asthma. Twelve of the individuals with chronic bronchitis were current or former smokers, and two asthmatics were former smokers.

The second group comprised 10 asthmatic subjects, in whom airway narrowing (i.e. a (mean $\pm \mathrm{SD})$ decrease of FEV1 of $28 \pm 9 \%$ ) was induced by inhaling methacholine (MCh group). To enter the study, subjects had to be in a stable clinical condition, free of respiratory symptoms, and to have a control FEV $1>70 \%$ pred and an FEV1/FVC within the normal range. One subject was a current smoker.

At the time of the study, none of the subjects was taking drugs other than short-acting inhaled $\beta_{2}$-agonists, which were withdrawn at least $8 \mathrm{~h}$ before the study.

\section{Lung function measurements}

Thoracic gas volume was measured with the subject sitting in a constant-volume body plethysmograph (Jaeger, Würzburg, Germany) and panting slowly against a closed shutter at end-tidal expiration. After opening the shutter, expiratory reserve volume and inspiratory $\mathrm{VC}$ were measured, thus allowing TLC to be calculated.

Flow was measured at the mouth by a screen-type heated pneumotachograph linear up to $16 \mathrm{~L} \cdot \mathrm{s}^{-1}$, coupled to a differential pressure transducer (Jaeger, Würzburg, Germany). The flow meter system was calibrated daily over the relevant flow range using a $1 \mathrm{~L}$ syringe driven manually. Appropriate corrections for temperature and gas viscosity [2] were applied. After careful correction for drift, by manually regulating a potentiometer, inspired and expired volumes were obtained by integration of the flow signal and recorded as spirogram tracings. Flow and volume signals were stored in a short-time memory screen, and then plotted slowly on an XY recorder (LY 1400 Linseis, Selb, Germany) as flow-volume curves. FEV1 was calculated according to the recommendations of the ATS.

Four VC measurements were obtained with two different sets of manoeuvres (fig. 1) performed in random order. The set of slow manoeuvres consisted of a slow expiration from end-tidal inspiration to RV followed by a fast inspiration to TLC (thus defining FIVCse) and, without breathholding, by a slow expiration to RV (thus

\section{a) Slow manoeuvres}

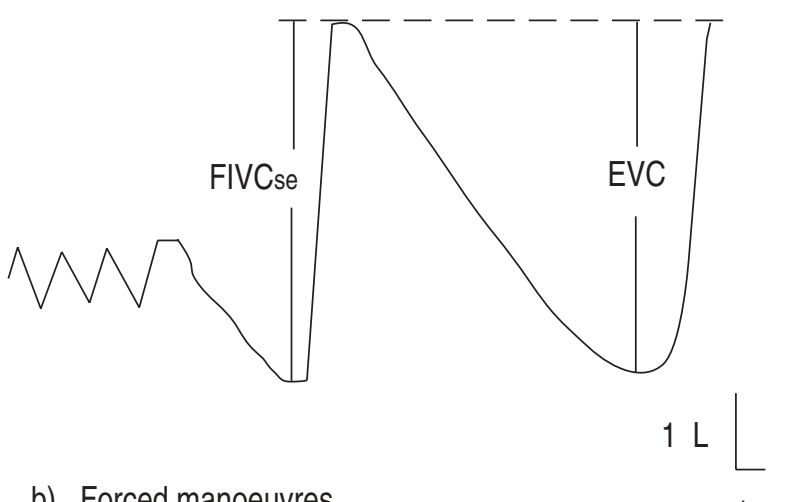

b) Forced manoeuvres

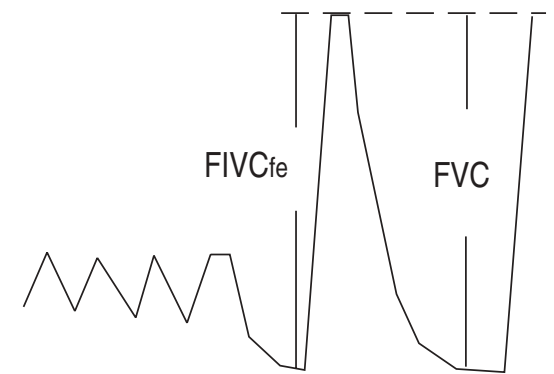

Fig. 1. - Diagrammatic representation of the manoeuvres used to measure vital capacities. a) FIVCse: forced inspiratory vital capacity after a slow expiration from end-tidal inspiration to residual volume (RV); EVC: slow expiratory vital capacity: b) FIVCfe: forced inspiratory vital capacity after a forced expiration from end-tidal inspiration to RV; FVC: forced expiratory vital capacity. Expiratory flow during FIVCse and EVC manoeuvres was $<0.5 \mathrm{~L} \cdot \mathrm{s}^{-1}$.

defining EVC). Expiratory flows were maintained $<0.5$ $\mathrm{L} \cdot \mathrm{s}^{-1}$. The set of forced manoeuvres consisted of a forced expiration from end-tidal inspiration to RV followed by a fast inspiration to TLC (thus defining FIVCfe) and, without breathholding, a forced expiration to RV (thus defining FVC). A deep breath to TLC was taken after completion of each set of manoeuvres to check for drift.

An interval of at least 2 min was allowed between the sets of slow and fast manoeuvres. In the CAO group, three sets of repeatable slow and fast manoeuvres were always recorded. In the MCh group, three sets of manoeuvres were recorded at control, but only one set after inhaling saline or $\mathrm{MCh}$.

Expiratory manoeuvres not showing a gradual decrease of volume change near RV or forced expiratory manoeuvres without a sharp peak flow were discarded. Inspiratory VCs (FIVCse and FIVCfe) were calculated on the spirogram as the volume between RV obtained after the partial expiratory manoeuvre and the subsequent TLC. Expiratory VCs (EVC and FVC) were calculated on the spirogram as the distance between the TLC and RV attained after the maximal expiratory manoeuvre.

\section{Methacholine inhalation challenge}

Aerosols were generated by using a dosimeter (MEFAR, Brescia, Italy), which delivers particles with an aerodynamic mass median diameter of 1.53-1.61 $\mu \mathrm{m}$. During quiet tidal inspiration, a breath-activated solenoid valve 
remained open for $1 \mathrm{~s}$, delivering $5 \mu \mathrm{L}$ of solution. Lung function measurements were obtained starting approximately 2 min after each aerosol inhalation. Saline solution $(0.9 \%)$ was inhaled and the FEV1 control value was determined. MCh was then administered in doubling doses, starting from $0.02 \mathrm{mg}$, until the FEV1 had decreased by $20 \%$ or more from control. Subjects were given a $\beta_{2}$-agonist to inhale after the challenge and remained in the laboratory until the FEV1 had returned to within $10 \%$ of the control value. None of the subjects experienced discomfort during the challenges.

\section{Statistical analysis}

Data are expressed as mean \pm standard deviation (SD). Deviations from predicted values for FEV1, FVC, TLC and RV are expressed as standardized residuals (SR) [2]. For comparison between and within groups, analysis of variance (ANOVA) with Duncan post-hoc test, unpaired t-test, and Pearson's correlation coefficient were used. A p-value less than 0.05 was considered statistically significant.

\section{Results}

Inhalation of the constric tor agent in the MCh group allowed comparison with the CAO group at similar values of FEV1 expressed as SRs (-2.3 \pm 1.02 vs -2.0 \pm 1.4$)$.

The VC values obtained with the different manoeuvres in the CAO group at control and in the MCh group during induced bronchoconstriction are presented in table 2 . There was no significant difference between groups, but highly significant differences $(\mathrm{p}<0.001)$ in various measurements of VC within groups, and also a highly significant interaction between groups and manoeuvres $(\mathrm{p}<$ $0.001)$. This indicates that the effect of the manoeuvres used to obtain VC was different in the two groups.

Within the CAO group, FIVCfe $(3.83 \pm 0.98 \mathrm{~L})$ was significantly larger than FVC $(3.75 \pm 1.03 \mathrm{~L} ; \mathrm{p}<0.01)$ but less

Table 2. - Comparison between lung function in CAO group and during bronchoconstriction in MCh group

\begin{tabular}{|c|c|c|c|}
\hline & O group & $\begin{array}{l}\mathrm{p} \text {-value } \\
\text { (between } \\
\text { groups) }\end{array}$ & $\begin{array}{l}\text { MCh group } \\
\text { (end-point) }\end{array}$ \\
\hline \multirow{2}{*}{$\begin{array}{ll}\text { FEV1 } & \text { L } \\
& \text { SR }\end{array}$} & $2.31 \pm 0.86$ & NS & $2.73 \pm 0.81$ \\
\hline & $-2.0 \pm 1.4$ & NS & $-2.3 \pm 1.02$ \\
\hline \multirow{2}{*}{$\begin{array}{l}\text { FEV } 1 / \text { FVC } \% \\
\text { FVC L }\end{array}$} & $60 \pm 13$ & NS & $65 \pm 8$ \\
\hline & $3.75 \pm 1.03$ & NS & $4.16 \pm 0.94$ \\
\hline \multirow{2}{*}{\begin{tabular}{l}
\multicolumn{2}{c}{ SR } \\
FIVCse $L$
\end{tabular}} & $-0.4 \pm 1.2$ & NS & $0.3 \pm 0.2$ \\
\hline & $4.03 \pm 0.91 * * *$ & NS & $3.99 \pm 0.89$ \\
\hline EVC L & $3.93 \pm 0.99 *$ & NS & $4.19 \pm 0.89$ \\
\hline FIVCfe $\mathrm{L}$ & $3.83 \pm 0.98 * *+$ & NS & $3.76 \pm 0.81 * *$ \\
\hline
\end{tabular}

Data are presented as mean \pm SD. FIVCse: forced inspiratory vital capacity after a slow expiration from end-tidal inspiration to residual volume (RV); EVC: slow expiratory vital capacity; FIVCfe: forced inspiratory vital capacity after a forced expiration from end-tidal inspiration to RV. For further definitions see legend to table 1 . FEV 1 and $\mathrm{FEV}_{1} / \mathrm{FVC}$ were compared between groups by unpaired t-test. FIVCse, EVC, FIVCfe and FVC were compared between and within groups by analysis of variance (ANOVA) and Duncan post-hoc test. *, **, ***: $\mathrm{p}<0.05, \mathrm{p}<0.01, \mathrm{p}<0.001$ vs $\mathrm{FVC}$, within the group; +: $\mathrm{p}<0.01$ vs FIVCse.
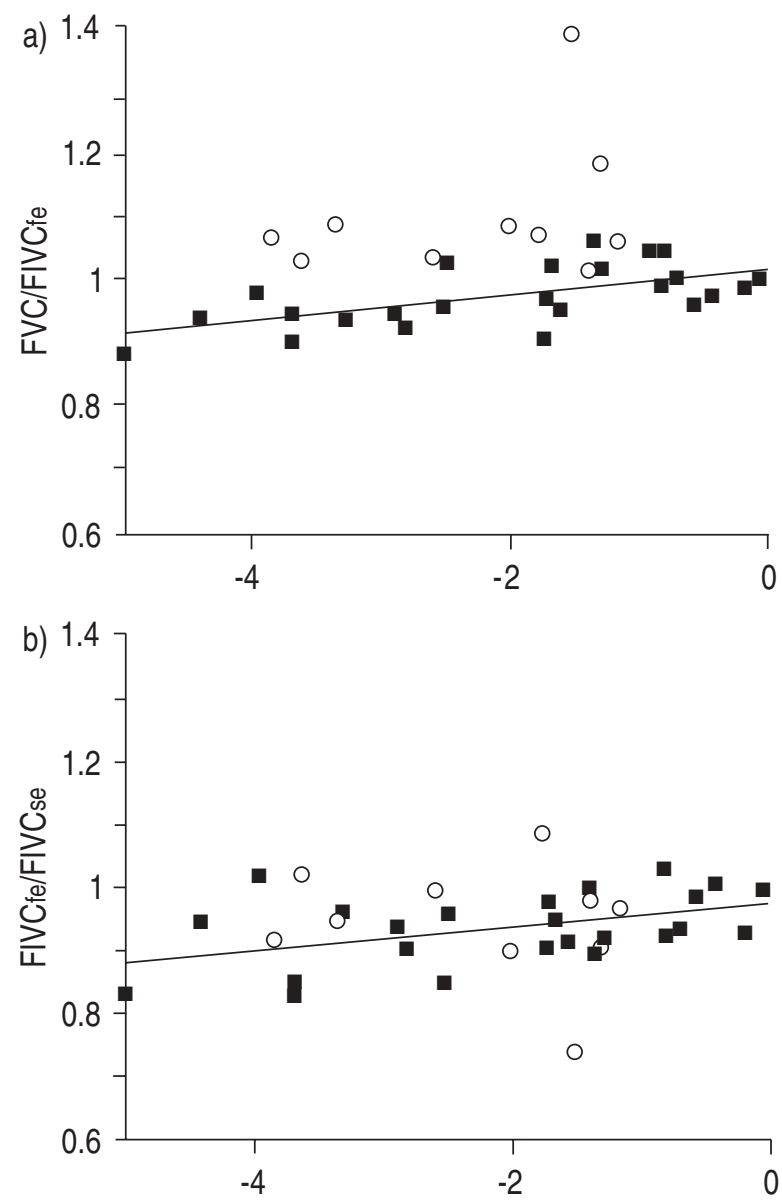

FEV1 SR

Fig. 2. - Relationships of: a) FVC/FIVCfe; and b) FIVCfe/FIVCse to forced expiratory volume in one second (FEV1) expressed as standardized residuals (SRs). For definitions see legend to figure 1. Both FVC/FIVCfe and FIVCfe/FIVCse correlate significantly with FEV1 $(\mathrm{r}=0.61 ; \mathrm{p}<0.005$, and $\mathrm{r}=0.49 ; \mathrm{p}<0.02$, respectively) only if subjects with induced bronchoconstriction (O) are excluded.

than FIVCse $(4.03 \pm 0.91 \mathrm{~L} ; \mathrm{p}<0.01)$. FVC was significantly less than FIVCse $(\mathrm{p}<0.001)$ and EVC $(3.93 \pm 0.99 \mathrm{~L}$; $\mathrm{p}<0.02)$. Within the MCh group, FVC $(4.16 \pm 0.94 \mathrm{~L})$ was significantly $(\mathrm{p}<0.01)$ larger than FIVCfe $(3.76 \pm 0.81 \mathrm{~L})$.

The effect of volume history can be inferred by comparing $\mathrm{FVC}$ to $\mathrm{FIVCfe}$, and the effect of flow history by comparing FIVCfe to FIVCse. The ratios of both FVC/ FIVCfe and FIVCfe/FIVCse correlated significantly ( $\mathrm{r}=$ $0.61 ; \mathrm{p}<0.005$ and $\mathrm{r}=0.49 ; \mathrm{p}<0.02$, respectively) with the FEV1 (expressed as SRs) in the CAO group but not in the MCh group (fig. 2).

\section{Discussion}

The main findings of this study are that: 1) the magnitude of VC depends both on the type of manoeuvre (expiratory or inspiratory) and the velocity of expiration to RV; and 2) these effects of volume and flow histories may differ in acute and chronic airway obstruction.

\section{Comments on methodology}

In this study, VCs were measured by integration of airflow measured at the mouth. Therefore, any effect of 
thoracic gas compression during expiration was not taken into account. Although gas compression affects flowvolume curves and FEV1, it should affect FVC only to the extent that there is gas compression at RV. This would cause thoracic gas volume excursion to exceed expired gas volume. As all VC manoeuvres either began or ended with maximal expiratory efforts, there is no reason to suspect that intrathoracic gas compression at RV would systematically bias the results.

The subjects were asked to sustain expiratory efforts for as long as they could. Therefore, the time for expiration was similar with all manoeuvres. This would have allowed more time for expiring at low lung volumes during forced than during slow manoeuvres. If $\mathrm{RV}$ was determined by the subjects' inability to sustain a forceful exhalation long enough to allow slow lung units to empty, the FVC would have been systematically greater than EVC. This was not the case in either group, as FVC was similar to EVC in the MCh group and smaller than EVC in the CAO group.

Carbon dioxide output is likely to exceed oxygen uptake during the time necessary to complete a VC manoeuvre [14]. This would result in expiratory VCs exceeding inspiratory VCs by $50-150 \mathrm{~mL}$. Therefore, only the difference between FVC and FIVCfe in the MCh group could be explained partly by the effect of gas exchange. Conversely, the differences between the expiratory and inspiratory VCs in CAO group would be slightly underestimated.

In the ensuing discussion, TLC will be assumed to be similar for all manoeuvres. We think this is reasonable because, although the initial expirations to RV prior to the maximal inspirations were initiated at different rates, they both ended with prolonged efforts near RV. If the difference between FIVCfe and FIVCse (significant in the CAO group, but not in the MCh group) had been due to differences in TLC rather than RV, FVC and EVC would also have been systematically different. This was the case in the CAO group but not in the MCh group.

\section{Comments on results}

From a physiological point of view, the magnitude of VC depends on the determinants of TLC and RV. In the absence of gross abnormalities in chest wall and inspiratory muscle mechanics, TLC is determined primarily by the lung elastic recoil [15]. In young healthy subjects, RV is determined primarily by static factors, i.e. the elastic recoil of the chest wall and the pressure of the expiratory muscles [3]. In middle-aged subjects or in the presence of airway obstruction, RV is also determined by dynamic factors, such as expiratory flow limitation [3] and airway closure [4]. Therefore, VC reflects parenchymal properties in normal individuals, but also airway properties in obstructed patients.

In clinical settings, VC is generally measured as a maximal inspiration from RV to TLC, or a slow or a forced maximal expiration from TLC to RV. Although inspiratory and expiratory manoeuvres are thought to give comparable results [16], it has been observed that VC values measured with a single expiration may be less than the sum of inspiratory capacity and expiratory reserve volume measured separately in some emphysematous subjects $[17,18]$. The reason for this discrepancy was unclear, but the effect of the deep inhalation to TLC on airway calibre when VC was measured as maximal expiration might have played a role. Indeed, it was recently shown that airway calibre is decreased after a full inflation in some patients with CAO [7]. Conversely, during induced bronchoconstriction, a full inflation increases airway calibre and reduces $\mathrm{RV}$ after a forced expiratory manoeuvre [11]. Consistent with this finding, the FVC in this study was larger than FIVCfe during $\mathrm{MCh}$-induced bronchoconstriction, but smaller in $\mathrm{CAO}$. Furthermore, the more severe the $\mathrm{CAO}$ the greater the difference between FVC and FIVCfe.

The mechanisms by which deep inhalation increases FVC may be the same as those thought to increase airway calibre. Because airway and lung parenchyma are interdependent, differences in hysteretical properties between the two may determine transient changes in airway calibre during inspiration and expiration. If airway hysteresis exceeds parenchymal hysteresis, then the airway calibre at a given lung volume is larger during expiration than during inspiration [5]. If flow limitation is a determinant of RV during bronchoconstriction, then an increase in airway calibre after deep inhalation may decrease RV, thus increasing FVC.

Although it has been reported that FVC may be similar to slow expiratory VC even in obstructed patients [15], some of the prediction equations currently used in pulmonary function testing [2] give FVC values that are slightly less $(\sim 5 \%)$ than slow VC values. Comparison of FVC with EVC is, however, complicated, because it depends on the combined effects of flow and volume history. Increasing the expiratory flow to RV resulted in a significant decrease in VC in the CAO group, depending on the severity of bronchial obstruction. However, the pattern was inconsistent in the MCh group. The mechanisms by which high flow decreases VC is not clear. One hypothesis is that an increase in airflow may increase viscous pressure losses within narrowed peripheral airways, thus causing the transmural pressure to be less and airway closure to occur at somewhat higher lung volume. Therefore, the greater the bronchoconstriction, the lower the VC after forced expiration, which would be in keeping with the correlation between the ratio of FIVCfe to FIVCse and FEV1 in the CAO group. The reason why this effect was inconsistent in the MCh group could be that MCh does not cause peripheral airway narrowing in all asthmatic subjects [10].

In conclusion, the results of this study are consistent with volume and flow histories together being determinants of the size of vital capacity. The effect of volume history on vital capacity seems to be related to the direction and the magnitude of the change induced by deep inhalation on airway calibre, and may depend on the interdependence between airways and lung parenchyma. The effect of flow history may be the result of changes in transmural pressure. An important practical implication of this study is that for vital capacity values to be comparable (either between or within subjects) they need to be obtained by the same manoeuvre.

\section{References}

1. Standardization of spirometry: 1987 update. Am Rev Respir Dis 1987; 136: 1285-1298. 
2. Quanjer PhH, Tammeling GJ, Cotes JE, Pedersen OF, Peslin R, Yernault J-C. Lung volumes and forced ventilatory flows. Report Working Party, "Standardization of Lung Function Tests". European Coal and Steel Community. Official statement of the European Respiratory Society. Eur Respir J 1993; 6 (Suppl. 16): 5-40.

3. Leith DE, Mead J. Mechanisms determining residual volume of the lungs in normal subjects. J Appl Physiol 1967; 23: 221-227.

4. Sutherland PW, Katsura T, Milic-Emili J. Previous volume history of the lung and regional distribution of gas. J Appl Physiol 1968; 25: 566-574.

5. Froeb HF, Mead J. Relative hysteresis of the dead space and lung in vivo. J Appl Physiol 1968; 25: 244-248.

6. Burns CB, Taylor WR, Ingram RH. Effects of deep inhalation in asthma: relative airway and parenchymal hysteresis. J Appl Physiol 1985; 59: 1590-1596.

7. Fairshter RD. Airway hysteresis in normal subjects and individuals with chronic airflow obstruction. $J$ Appl Physiol 1985; 58: 1505-1510.

8. Lim TK, Pride NB, Ingram RH. Effects of volume history during spontaneous and acutely induced airflow obstruction in asthma. Am Rev Respir Dis 1987; 135: 591-596.

9. Pellegrino R, Violante B, Crimi E, Brusasco V. Effects of aerosol methacholine and histamine on airways and lung parenchyma in healthy humans. J Appl Physiol 1993; 74: 2681-2686.

10. Brusasco V, Pellegrino R, Violante B, Crimi E. Relationship between quasistatic pulmonary hysteresis and maximal airway narrowing in humans. $J$ Appl Physiol 1992; 72: 2075-2080.

11. Pellegrino R, Violante B, Selleri R, Brusasco V. Changes in residual volume during induced bronchoconstriction in healthy and asthmatic subjects. Am J Respir Crit Care Med 1994; 150: 363-368.

12. Skloot G, Permutt S, Togias A. Deep inspiration affects airway caliber and airway closure in asthma. Am Rev Respir Dis 1993; 147: A257.

13. American Thoracic Society. Chronic bronchitis, asthma and pulmonary emphysema. Am Rev Respir Dis 1962; 84: 762-768.

14. DeGroodt EG, Quanjer PH, Wise ME. Influence of external resistance and minor flow variations on single breath nitrogen test and residual volume. Bull Eur Physiopathol Respir 1983; 19: 267-272.

15. Anthonisen NR. Tests of mechanical function. In: Macklem PT, Mead J, eds. Handbook of Physiology. Section 3, Vol. III, Part 2. The Respiratory System: Mechanics of Breathing. Bethesda, MD, American Physiological Society, 1986; pp. 753-784.

16. Cournand A, Richards DW, Darling RC. Graphic tracings of respiration in study of pulmonary disease. Am Rev Tuber 1939; 44: 123-172.

17. Clarke SW, Jones JG, Glaister DH. Changes in pulmonary ventilation in different postures. Clin Sci 1969; 37: 357-369.

18. Hansen ALM, Pedersen OF, Lyager S, Næraa N. Metodebetingede forskelle i vitalkapacitet. Ugeskr Larger 1983; 145: 2752-2756. 